\title{
Two-loop QCD corrections to vector boson pair production at the LHC
}

\author{
Lorenzo Tancredi* \\ Institut für Theoretische Teilchenphysik, KIT, 76128 Karlsruhe, Germany \\ E-mail: lorenzo.tancredi@kit.edu
}

\section{Thomas Gehrmann}

Department of Physics, University of Zürich, Winterthurerstrasse 190, CH-8057 Zürich, Switzerland

E-mail: thomas.gehrmannduzh.ch

\section{Andreas von Manteuffel}

PRISMA Cluster of Excellence, Institute of Physics, Johannes Gutenberg University, 55099 Mainz, Germany

E-mail: manteuffelduni-mainz.de

We report on the calculation of the two-loop QCD helicity amplitudes for the production of pairs of off-shell electroweak vector bosons in the $q \bar{q}^{\prime}$ and $g g$ channels. These amplitudes were the last missing ingredient required for the evaluation of the NNLO QCD corrections to vector boson pair production at the LHC.

12th International Symposium on Radiative Corrections (Radcor 2015) and LoopFest XIV (Radiative Corrections for the LHC and Future Colliders)

15-19 June 2015

UCLA Department of Physics \& Astronomy Los Angeles, CA, USA

${ }^{*}$ Speaker. 


\section{Introduction and motivation}

The study of vector boson pair production at the LHC provides some of the the most interesting observables both for Standard Model and Beyond Standard Model physics. Indeed, the long sought Higgs boson was recently discovered at the LHC mainly due its decays to pairs of electroweak vector bosons [1,2]. Moreover, precise theoretical predictions for these processes will be important to determine its quantum numbers, its couplings and its width [3,4]. Finally, vector boson pair production plays also a crucial role in new physics searches, as it allows to put constraints on anomalous gauge couplings and constitutes the natural background for many searches for new resonances.

Production of electroweak gauge boson pairs at the LHC occurs mainly through quark-antiquark annihilation, $q \bar{q}^{\prime} \rightarrow V_{1} V_{2}$, but a sizable contribution comes also from the gluon-fusion channel $g g \rightarrow V_{1} V_{2}$ [5-7]. The latter starts at one loop and therefore contributes formally only from NNLO onwards to the full partonic process $p p \rightarrow V_{1} V_{2}$. Nevertheless, being effectively a LO process, it is expected to suffer from rather large theoretical uncertainties, poorly estimated by simple scale variation. Moreover, as for the kinematically similar $g g \rightarrow H$ process, we expect large QCD radiative corrections $(\approx \mathscr{O}(100 \%)$ ) due to the large color charge of the $g g$ initial state [8]. Finally, it is well known that, depending on the final state and on the cuts applied, the $g g$ channel can amount to around $10 \%$ or more of the total cross section $[9,10]$. It becomes therefore clear that, in order to reduce the theoretical uncertainty to $\approx 5 \%$, as required to match the expected experimental precision to be reached by the LHC Run II, the calculation of the NNLO corrections to the quark-antiquark channel must be supplemented by the corresponding NLO corrections to the gluon-fusion channel.

At NNLO, differential predictions for the $q \bar{q}^{\prime}$ channel have been computed for $\gamma \gamma, Z \gamma, W^{ \pm} \gamma$ and $Z Z$ [9-13], while only the fully inclusive cross-section [14] and the correspondingly normalized resummed $p_{T}$ spectra [15] are currently known for $W^{+} W^{-}$final states. For $\gamma \gamma$ the NLO corrections to the $g g$ channel have been known for a long time [16], while recently also those for $\mathrm{ZZ}$ and $W^{+} W^{-}$final states have been computed [17,18], confirming that they are crucial to provide a reliable estimate of the theoretical uncertainties. These calculations have been made possible by the recent evaluation of the two-loop massless QCD corrections to the corresponding helicity amplitudes [19-24]. In next section we will report in particular on the computation of the twoloop helicity amplitudes for the production of two off-shell electroweak vector bosons as described in $[22,24]$.

\section{The calculation of the two-loop helicity amplitudes}

At variance with one-loop, two-loop calculations are far from being automatized and constitute often the bottleneck to complete NNLO calculations. The by now standard approach to two-loop calculations is based on the use of integration-by-parts identities [25,26] and the Laporta algorithm [27], in order to reduce the thousands of scalar integrals appearing in a typical $2 \rightarrow 2$ process to a small subset of master integrals (MIs). The computation of the helicity amplitudes for $q \bar{q}^{\prime} \rightarrow V_{1} V_{2}$ and $g g \rightarrow V_{1} V_{2}$ in particular proceeds as follows. We consider the two processes

$$
p\left(p_{1}\right)+p\left(p_{2}\right) \rightarrow V_{1}\left(p_{3}\right)+V_{2}\left(p_{4}\right), \quad \text { with } \quad p_{1}^{2}=p_{2}^{2}=0, \quad p_{3}^{2} \neq p_{4}^{2}, \quad p_{3}^{2}, p_{4}^{2} \neq 0
$$


where the $p\left(p_{i}\right)$ are either quarks or gluons, and define

$$
s=\left(p_{1}+p_{2}\right)^{2}, \quad t=\left(p_{1}-p_{3}\right)^{2}, \quad u=\left(p_{2}-p_{3}\right)^{2} .
$$

We start by considering the most general form for the scattering amplitudes allowed by Lorentz invariance and gauge invariance. Schematically for $q \bar{q}^{\prime} \rightarrow V_{1} V_{2}$ we find for the partonic current $S_{q \bar{q}^{\prime}}^{\mu v}$ stripped of all couplings

$$
\varepsilon_{3 \mu} \varepsilon_{4 v} S_{q \bar{q}^{\prime}}^{\mu v}=\varepsilon_{3 \mu} \varepsilon_{4 v} \sum_{j=1}^{10} A_{q \bar{q}^{\prime}}^{(j)} t_{j}^{\mu v}
$$

with

$$
\begin{array}{lll}
t_{1}^{\mu v}=\bar{u}\left(p_{2}\right) \not p_{3} u\left(p_{1}\right) p_{1}^{\mu} p_{1}^{v}, & t_{2}^{\mu v}=\bar{u}\left(p_{2}\right) \not p_{3} u\left(p_{1}\right) p_{1}^{\mu} p_{2}^{v}, & t_{3}^{\mu v}=\bar{u}\left(p_{2}\right) \not p_{3} u\left(p_{1}\right) p_{2}^{\mu} p_{1}^{v}, \\
t_{4}^{\mu v}=\bar{u}\left(p_{2}\right) \not p_{3} u\left(p_{1}\right) p_{2}^{\mu} p_{2}^{v}, & t_{5}^{\mu v}=\bar{u}\left(p_{2}\right) \gamma^{\mu} u\left(p_{1}\right) p_{1}^{v}, & t_{6}^{\mu v}=\bar{u}\left(p_{2}\right) \gamma^{\mu} u\left(p_{1}\right) p_{2}^{v}, \\
t_{7}^{\mu v}=\bar{u}\left(p_{2}\right) \gamma^{v} u\left(p_{1}\right) p_{1}^{\mu}, & t_{8}^{\mu v}=\bar{u}\left(p_{2}\right) \gamma^{v} u\left(p_{1}\right) p_{2}^{\mu}, & t_{9}^{\mu v}=\bar{u}\left(p_{2}\right) \gamma^{\mu} \not p_{3} \gamma^{v} u\left(p_{1}\right), \\
t_{10}^{\mu v}=\bar{u}\left(p_{2}\right) \gamma^{v} \not p_{3} \gamma^{\mu} u\left(p_{1}\right) . &
\end{array}
$$

Similarly, for the case $g g \rightarrow V_{1} V_{2}$ we have for the partonic current $S_{g g}^{\mu \nu}$

$$
\varepsilon_{3 \mu} \varepsilon_{4 v} S_{g g}^{\mu v}=\varepsilon_{3 \mu} \varepsilon_{4 v} \sum_{j=1}^{20} A_{g g}^{(j)} \tau_{j}^{\mu v}
$$

with

$$
\begin{aligned}
& \tau_{1}^{\mu v}=\varepsilon_{1} \cdot \varepsilon_{2} g^{\mu v}, \quad \tau_{2}^{\mu v}=\varepsilon_{1}^{\mu} \varepsilon_{2}^{v}, \quad \tau_{3}^{\mu v}=\varepsilon_{1}^{v} \varepsilon_{2}^{\mu}, \quad \tau_{4}^{\mu v}=\varepsilon_{1} \cdot \varepsilon_{2} p_{1}^{\mu} p_{1}^{v}, \\
& \tau_{5}^{\mu v}=\varepsilon_{1} \cdot \varepsilon_{2} p_{1}^{\mu} p_{2}^{v}, \quad \tau_{6}^{\mu v}=\varepsilon_{1} \cdot \varepsilon_{2} p_{2}^{\mu} p_{1}^{v}, \quad \tau_{7}^{\mu v}=\varepsilon_{1} \cdot \varepsilon_{2} p_{2}^{\mu} p_{2}^{v}, \quad \tau_{8}^{\mu v}=\varepsilon_{2} \cdot p_{3} \varepsilon_{1}^{\mu} p_{1}^{v}, \\
& \tau_{9}^{\mu v}=\varepsilon_{2} \cdot p_{3} \varepsilon_{1}^{\mu} p_{2}^{v}, \quad \tau_{10}^{\mu v}=\varepsilon_{2} \cdot p_{3} \varepsilon_{1}^{v} p_{1}^{\mu}, \quad \tau_{11}^{\mu v}=\varepsilon_{2} \cdot p_{3} \varepsilon_{1}^{v} p_{2}^{\mu}, \quad \tau_{12}^{\mu v}=\varepsilon_{1} \cdot p_{3} \varepsilon_{2}^{\mu} p_{1}^{v}, \\
& \tau_{13}^{\mu v}=\varepsilon_{1} \cdot p_{3} \varepsilon_{2}^{\mu} p_{2}^{v}, \quad \tau_{14}^{\mu v}=\varepsilon_{1} \cdot p_{3} \varepsilon_{2}^{v} p_{1}^{\mu}, \quad \tau_{15}^{\mu v}=\varepsilon_{1} \cdot p_{3} \varepsilon_{2}^{v} p_{2}^{\mu}, \quad \tau_{16}^{\mu v}=\varepsilon_{1} \cdot p_{3} \varepsilon_{2} \cdot p_{3} g^{\mu v}, \\
& \tau_{17}^{\mu v}=\varepsilon_{1} \cdot p_{3} \varepsilon_{2} \cdot p_{3} p_{1}^{\mu} p_{1}^{v}, \quad \tau_{18}^{\mu v}=\varepsilon_{1} \cdot p_{3} \varepsilon_{2} \cdot p_{3} p_{1}^{\mu} p_{2}^{v}, \\
& \tau_{19}^{\mu v}=\varepsilon_{1} \cdot p_{3} \varepsilon_{2} \cdot p_{3} p_{2}^{\mu} p_{1}^{v}, \quad \tau_{20}^{\mu v}=\varepsilon_{1} \cdot p_{3} \varepsilon_{2} \cdot p_{3} p_{2}^{\mu} p_{2}^{v} \text {. }
\end{aligned}
$$

We imposed everywhere $\varepsilon_{j} \cdot p_{j}=0$ for $j=1,2,3,4$ together with $\varepsilon_{1} \cdot p_{2}=\varepsilon_{2} \cdot p_{1}=0$. The coefficients $A_{X}^{(j)}$, for the two different initial states $X=q \bar{q}^{\prime}, g g$, are scalar coefficients and depend on the perturbative order of the calculation. Given the tensors (2.1), (2.3), we proceed by defining projection operators which, once applied to the amplitude as described below, extract the scalar coefficients $A_{X}^{(j)}$ for the given initial state $X$ :

$$
P_{q q, k}^{\mu v}=\sum_{j=1}^{10} a_{q q, k}^{(j)}\left(t_{j}^{\mu v}\right)^{\dagger}, \quad P_{g g, k}^{\mu v}=\sum_{j=1}^{20} a_{g g, k}^{(j)}\left(\tau_{j}^{\mu v}\right)^{\dagger}
$$

defined such that

$$
\sum_{p o l} P_{X, k}^{\mu v}\left(g_{\mu \rho}-\frac{p_{3 \mu} p_{3 \rho}}{p_{3}^{2}}\right)\left(g_{v \sigma}-\frac{p_{4 v} p_{4 \sigma}}{p_{4}^{2}}\right) S_{X}^{\rho \sigma}=A_{X}^{(k)}
$$


In (2.5) we require explicitly transversality for the massive vector bosons, which is a consequence of having imposed $\varepsilon_{3} \cdot p_{3}=\varepsilon_{4} \cdot p_{4}=0$ on (2.1) and (2.3). We note here that the tensors and the projectors are defined in $d$ continuous dimensions.

We then proceed by generating the relevant Feynman diagrams with QGRAF [28], apply on them the projectors (2.4), and perform the sum over polarizations and the Dirac algebra with FORM [29]. In this way we can write the scalar coefficients $A_{X}^{(k)}$ as linear combinations of scalar Feynman integrals, i.e. integrals whose numerators contain at most scalar products of internal and external momenta. The latter can be collected into three independent integral families, for which we generated and solved integration-by-parts identities using the automated code Reduze 2 [30-33]. This allowed us to reduce all integrals to the master integrals for massless four-point functions with two off-shell legs. Their calculation had remained for a long time an outstanding task, which was recently solved by different groups independently [22,34-38]. The calculation is based on the differential equation method [39-41] augmented by the choice of a canonical basis [42]. This makes the analytic structure of the solution evident and allows to greatly simplify their integration in terms of multiple polylogarithms [43-45].

In order to integrate the differential equations we employ the parametrization

$$
\begin{array}{ll}
s=\bar{m}^{2}(1+\bar{x})^{2}, & p_{3}^{2}=\bar{m}^{2} \bar{x}^{2}\left(1-\bar{y}^{2}\right), \\
t=-\bar{m}^{2} \bar{x}((1+\bar{y})(1+\bar{x} \bar{y})-2 \bar{z} \bar{y}(1+\bar{x})), & p_{4}^{2}=\bar{m}^{2}\left(1-\bar{x}^{2} \bar{y}^{2}\right),
\end{array}
$$

which rationalizes the Källen function for the two-body decay and allows us to write all master integrals in terms of multiple polylogarithms with the following alphabet

$$
\begin{aligned}
\left\{\bar{l}_{1}, \ldots, \bar{l}_{20}\right\}=\{ & 2, \bar{x}, 1+\bar{x}, 1-\bar{y}, \bar{y}, 1+\bar{y}, 1-\bar{x} \bar{y}, 1+\bar{x} \bar{y}, 1-\bar{z}, \bar{z}, \\
& 1+\bar{y}-2 \bar{y} \bar{z}, 1-\bar{y}+2 \bar{y} \bar{z}, 1+\bar{x} \bar{y}-2 \bar{x} \bar{y} \bar{z}, 1-\bar{x} \bar{y}+2 \bar{x} \bar{y} \bar{z}, \\
& 1+\bar{y}+\bar{x} \bar{y}+\bar{x} \bar{y}^{2}-2 \bar{y} \bar{z}-2 \bar{x} \bar{y} \bar{z}, 1+\bar{y}-\bar{x} \bar{y}-\bar{x} \bar{y}^{2}-2 \bar{y} \bar{z}+2 \bar{x} \bar{y} \bar{z}, \\
& 1-\bar{y}-\bar{x} \bar{y}+\bar{x} \bar{y}^{2}+2 \bar{y} \bar{z}+2 \bar{x} \bar{y} \bar{z}, 1-\bar{y}+\bar{x} \bar{y}-\bar{x} \bar{y}^{2}+2 \bar{y} \bar{z}-2 \bar{x} \bar{z} \bar{z}, \\
& 1-2 \bar{y}-\bar{x} \bar{y}+\bar{y}^{2}+2 \bar{x}^{2}-\bar{x} \bar{y}^{3}+4 \bar{y} \bar{z}+2 \bar{x} \bar{y} \bar{z}+2 \bar{x} \bar{y}^{3} \bar{z}, \\
& \left.1-\bar{y}-2 \bar{x} \bar{y}+2 \bar{x} \bar{y}^{2}+\bar{x}^{2} \bar{y}^{2}-\bar{x}^{2} \bar{y}^{3}+2 \bar{y} \bar{z}+4 \bar{x} \bar{y} \bar{z}+2 \bar{x}^{2} \bar{y}^{3} \bar{z}\right\},
\end{aligned}
$$

providing in this way an explicit analytical solution for the two-loop corrections to the coefficients $A_{X}^{(j)}$. The expressions obtained in this way, nevertheless, are not yet suitable for phenomenological applications. First of all we need to take into account the decays of the two vector bosons into massless leptons, assuming 4-dimensional external states and fixing their helicities. It is easy to see that in both cases for $X=g g$ and $X=q \bar{q}^{\prime}$, there are only two independent helicity configurations which can be conveniently written down using the spinor helicity formalism. As exemplification we consider the gluon-fusion channel

$$
g\left(p_{1}\right)+g\left(p_{2}\right) \rightarrow V_{1}\left(p_{3}\right)+V_{1}\left(p_{4}\right) \rightarrow l\left(p_{5}\right)+\bar{l}\left(p_{6}\right)+l\left(p_{7}\right)+\bar{l}\left(p_{8}\right) .
$$

We choose left-handed leptonic decay currents ${ }^{1}$ and obtain for the two independent helicity configurations

\footnotetext{
${ }^{1}$ Note that the corresponding right-handed ones can be obtained exchanging the lepton-antilepton momenta $p_{5} \leftrightarrow$ $p_{6}, p_{7} \leftrightarrow p_{8}$
} 


$$
\begin{aligned}
\mathrm{M}_{\lambda_{1} \lambda_{2} L L}\left(p_{1}, p_{2} ; p_{5}, p_{6}, p_{7}, p_{8}\right) & =C_{\lambda_{1} \lambda_{2}}\left[[ 2 \not p _ { 3 } 1 \rangle \left\{E_{1}^{\lambda_{1} \lambda_{2}}\langle 57\rangle[68]\right.\right. \\
& +E_{2}^{\lambda_{1} \lambda_{2}}\langle 15\rangle\langle 17\rangle[16][18]+E_{3}^{\lambda_{1} \lambda_{2}}\langle 15\rangle\langle 27\rangle[16][28] \\
& \left.+E_{4}^{\lambda_{1} \lambda_{2}}\langle 25\rangle\langle 17\rangle[26][18]+E_{5}^{\lambda_{1} \lambda_{2}}\langle 25\rangle\langle 27\rangle[26][28]\right\} \\
& +E_{6}^{\lambda_{1} \lambda_{2}}\langle 15\rangle\langle 17\rangle[16][28]+E_{7}^{\lambda_{1} \lambda_{2}}\langle 15\rangle\langle 17\rangle[26][18] \\
& \left.+E_{8}^{\lambda_{1} \lambda_{2}}\langle 15\rangle\langle 27\rangle[26][28]+E_{9}^{\lambda_{1} \lambda_{2}}\langle 25\rangle\langle 17\rangle[26][28]\right],
\end{aligned}
$$

with

$$
C_{L L}=\left[1 \not p_{3} 2\right\rangle \frac{\langle 12\rangle}{[12]}, \quad C_{L R}=\left[2 \not p_{3} 1\right\rangle,
$$

where the coefficients $E_{j}^{\lambda_{1} \lambda_{2}}$ are linear combinations of the $A_{X}^{(j)}$. Still the amplitudes are not in optimal form for numerical evaluation. The scalar coefficients $E_{j}^{\lambda_{1} \lambda_{2}}$ are given by large combinations of rational prefactors and complicated multiple polylogarithms. While this does not constitute a problem in principle, it does in practice, as it renders the numerical evaluation of the result using [46] very slow and unstable. To fix this issue we have used a private implementation of the algorithm of $[47,48]$ in order to rewrite our result in terms optimized functions. In particular we remapped our result to real valued $\log \left(l_{j}\right), \mathrm{Li}_{n}\left(R_{i}\right)$ and $\mathrm{Li}_{2,2}\left(R_{i}, S_{j}\right)$, choosing

$$
l_{j} \geq 0, \quad\left|R_{i}\right| \leq 1, \quad\left|R_{i} S_{j}\right|<1,
$$

such that all functions have convergent series expansions over the whole physical phase space and their numerical evaluation becomes therefore much simpler. In this way we obtain very fast and stable amplitudes, which can be evaluated in double, quadruple and arbitrary precision, over the whole phase space, allowing for a reliable precision control system. Limiting ourselves to double precision, we find for a typical phase space point in the bulk of the phase space an evaluation time of $\mathscr{O}(125 \mathrm{~ms})$ for $X=q \bar{q}^{\prime}$ and $\mathscr{O}(600 \mathrm{~ms})$ for $X=g g$ on a single CPU core ${ }^{2}$.

\section{Conclusions and outlook}

In this contribution we described the computation of the two-loop QCD corrections to the helicity amplitudes for the production of two massive vector bosons in $q \bar{q}^{\prime}$ annihilation and $g g$ fusion. These amplitudes were the last missing pieces needed to complete the calculation of the NNLO QCD corrections to vector boson pair production at LHC. The result has been optimized for fast and precise numerical evaluation making their use suitable for phenomenological studies. This opened up the path towards precision phenomenology in gauge boson pair production and improvements of the background predictions for Higgs boson studies and searches for physics beyond the Standard Model, as showed by the recent calculations $[9,10,17,18]$.

\footnotetext{
${ }^{2}$ The amplitudes are available as $\mathrm{C}++$ codes at http://vvamp.hepforge.org/.
} 


\section{References}

[1] ATLAS Collaboration, G. Aad et al., Observation of a new particle in the search for the Standard Model Higgs boson with the ATLAS detector at the LHC, Phys. Lett. $\mathbf{B 7 1 6}$ (2012) 1-29, [arXiv:1207.7214].

[2] CMS Collaboration, S. Chatrchyan et al., Observation of a new boson at a mass of $125 \mathrm{GeV}$ with the CMS experiment at the LHC, Phys. Lett. B716 (2012) 30-61, [arXiv: 1207 . 7235].

[3] N. Kauer and G. Passarino, Inadequacy of zero-width approximation for a light Higgs boson signal, JHEP 1208 (2012) 116, [arXiv: 1206.4803 ].

[4] F. Caola and K. Melnikov, Constraining the Higgs boson width with ZZ production at the LHC, Phys.Rev. D88 (2013) 054024, [arXiv:1307.4935].

[5] E. W. N. Glover and J. van der Bij, Z Boson Pair Production via Gluon Fusion, Nucl.Phys. B321 (1989) 561.

[6] E. N. Glover and J. van der Bij, Vector Boson Pair Production via Gluon Fusion, Phys.Lett. B219 (1989) 488.

[7] T. Binoth, M. Ciccolini, N. Kauer, and M. Kramer, Gluon-induced WW background to Higgs boson searches at the LHC, JHEP 0503 (2005) 065, [hep-ph/ 0503094 ].

[8] C. Anastasiou and K. Melnikov, Higgs boson production at hadron colliders in NNLO QCD, Nucl. Phys. B646 (2002) 220-256, [hep-ph/ 0207004 ].

[9] F. Cascioli, T. Gehrmann, M. Grazzini, S. Kallweit, P. Maierhöfer, A. von Manteuffel, S. Pozzorini, D. Rathlev, and L. Tancredi, ZZ production at hadron colliders in NNLO QCD, Phys.Lett. $\mathbf{B 7 3 5}$ (2014) 311-313, [arXiv:1405.2219].

[10] T. Gehrmann, M. Grazzini, S. Kallweit, P. Maierhöfer, A. von Manteuffel, S. Pozzorini, D. Rathlev, and L. Tancredi, $W^{+} W^{-}$Production at Hadron Colliders in Next to Next to Leading Order QCD, Phys.Rev.Lett. 113 (2014) 212001, [arXiv: 1408.5243 ].

[11] S. Catani, L. Cieri, D. de Florian, G. Ferrera, and M. Grazzini, Diphoton production at hadron colliders: a fully-differential QCD calculation at NNLO, Phys.Rev.Lett. 108 (2012) 072001, [arXiv:1110.2375].

[12] M. Grazzini, S. Kallweit, and D. Rathlev, ZZ production at the LHC: fiducial cross sections and distributions in NNLO QCD, Phys. Lett. B750 (2015) 407-410, [arXiv: 1507.06257 ].

[13] M. Grazzini, S. Kallweit, and D. Rathlev, W $\gamma$ and $Z \gamma$ production at the LHC in NNLO QCD, JHEP 07 (2015) 085, [arXiv:1504.01330].

[14] M. Grazzini, S. Kallweit, D. Rathlev, and A. Torre, Z $\gamma$ production at hadron colliders in NNLO QCD, Phys.Lett. B731 (2014) 204, [arXiv:1309. 7000 ].

[15] M. Grazzini, S. Kallweit, D. Rathlev, and M. Wiesemann, Transverse-momentum resummation for vector-boson pair production at NNLL+NNLO, JHEP 08 (2015) 154, [arXiv: 1507.02565 ].

[16] Z. Bern, L. J. Dixon, and C. Schmidt, Isolating a light Higgs boson from the diphoton background at the CERN LHC, Phys.Rev. D66 (2002) 074018, [hep-ph/ 0206194 ].

[17] F. Caola, K. Melnikov, R. Roentsch, and L. Tancredi, QCD corrections to ZZ production in gluon fusion at the LHC, Phys. Rev. D92 (2015), no. 9 094028, [arXiv: 1509.06734 ]. 
[18] F. Caola, K. Melnikov, R. Roentsch, and L. Tancredi, QCD corrections to $W^{+} W^{-}$production through gluon fusion, arXiv:1511.08617.

[19] T. Gehrmann and L. Tancredi, Two-loop QCD helicity amplitudes for $q \bar{q} \rightarrow W^{ \pm} \gamma$ and $q \bar{q} \rightarrow Z^{0} \gamma$, JHEP 1202 (2012) 004, [arXiv: 1112 .1531].

[20] T. Gehrmann, L. Tancredi, and E. Weihs, Two-loop QCD helicity amplitudes for $g \mathrm{~g} \rightarrow \mathrm{Z} g$ and $g g \rightarrow Z \gamma$, JHEP 1304 (2013) 101, [arXiv: 1302.2630].

[21] F. Caola, J. M. Henn, K. Melnikov, A. V. Smirnov, and V. A. Smirnov, Two-loop helicity amplitudes for the production of two off-shell electroweak bosons in quark-antiquark collisions, JHEP 11 (2014) 041, [arXiv:1408.6409].

[22] T. Gehrmann, A. von Manteuffel, and L. Tancredi, The two-loop helicity amplitudes for $q \bar{q}^{\prime} \rightarrow V_{1} V_{2} \rightarrow 4$ leptons, JHEP 09 (2015) 128, [arXiv: 1503.04812 ].

[23] F. Caola, J. M. Henn, K. Melnikov, A. V. Smirnov, and V. A. Smirnov, Two-loop helicity amplitudes for the production of two off-shell electroweak bosons in gluon fusion, JHEP 06 (2015) 129, [arXiv:1503.08759].

[24] A. von Manteuffel and L. Tancredi, The two-loop helicity amplitudes for $g g \rightarrow V_{1} V_{2} \rightarrow 4$ leptons, JHEP 06 (2015) 197, [arXiv: 1503 . 08835].

[25] K. Chetyrkin and F. Tkachov, Integration by Parts: The Algorithm to Calculate beta Functions in 4 Loops, Nucl.Phys. B192 (1981) 159-204.

[26] F. Tkachov, A Theorem on Analytical Calculability of Four Loop Renormalization Group Functions, Phys.Lett. B100 (1981) 65-68.

[27] S. Laporta, High precision calculation of multiloop Feynman integrals by difference equations, Int.J.Mod.Phys. A15 (2000) 5087-5159, [hep-ph/ 0102033 ].

[28] P. Nogueira, Automatic Feynman graph generation, J.Comput.Phys. 105 (1993) 279-289.

[29] J. Vermaseren, New features of FORM, math-ph/0010025.

[30] C. Studerus, Reduze-Feynman Integral Reduction in C++, Comput.Phys.Commun. 181 (2010) 1293-1300, [arXiv:0912.2546].

[31] A. von Manteuffel and C. Studerus, Reduze 2 - Distributed Feynman Integral Reduction, arXiv:1201.4330.

[32] R. Lewis, Computer Algebra System Fermat. http://www.bway.net/ lewis.

[33] C. W. Bauer, A. Frink, and R. Kreckel, Introduction to the GiNaC framework for symbolic computation within the C++ programming language, J.Symb.Comput. 33 (2002) 1-12, [CS/ 0004015$]$.

[34] T. Gehrmann, L. Tancredi, and E. Weihs, Two-loop master integrals for $q \bar{q} \rightarrow V V$ : the planar topologies, JHEP 1308 (2013) 070, [arXiv: 1306.6344 ].

[35] T. Gehrmann, A. von Manteuffel, L. Tancredi, and E. Weihs, The two-loop master integrals for $q \bar{q} \rightarrow V V$, JHEP 1406 (2014) 032, [arXiv : 1404 . 4853].

[36] J. M. Henn, K. Melnikov, and V. A. Smirnov, Two-loop planar master integrals for the production of off-shell vector bosons in hadron collisions, JHEP 1405 (2014) 090, [arXiv: 1402 . 7078 ].

[37] F. Caola, J. M. Henn, K. Melnikov, and V. A. Smirnov, Non-planar master integrals for the production of two off-shell vector bosons in collisions of massless partons, JHEP 1409 (2014) 043, [arXiv:1404.5590v2]. 
[38] C. G. Papadopoulos, D. Tommasini, and C. Wever, Two-loop Master Integrals with the Simplified Differential Equations approach, JHEP 1501 (2015) 072, [arXiv: 1409.6114$].$

[39] A. Kotikov, Differential equations method: New technique for massive Feynman diagrams calculation, Phys.Lett. B254 (1991) 158-164.

[40] E. Remiddi, Differential equations for Feynman graph amplitudes, Nuovo Cim. A110 (1997) 1435-1452, [hep-th/9711188].

[41] T. Gehrmann and E. Remiddi, Differential equations for two loop four point functions, Nucl.Phys. B580 (2000) 485-518, [hep-ph/9912329].

[42] J. M. Henn, Multiloop integrals in dimensional regularization made simple, Phys.Rev.Lett. 110 (2013) 251601, [arXiv:1304.1806].

[43] E. Remiddi and J. Vermaseren, Harmonic polylogarithms, Int.J.Mod.Phys. A15 (2000) 725-754, [hep-ph/9905237].

[44] T. Gehrmann and E. Remiddi, Two loop master integrals for $\gamma^{*} \rightarrow 3$ jets: The Planar topologies, Nucl.Phys. B601 (2001) 248-286, [hep-ph/ 0008287 ].

[45] A. Goncharov, Multiple polylogarithms and mixed Tate motives, math/ 0103059.

[46] J. Vollinga and S. Weinzierl, Numerical evaluation of multiple polylogarithms, Comput.Phys.Commun. 167 (2005) 177, [hep-ph/ 0410259 ].

[47] C. Duhr, H. Gangl, and J. R. Rhodes, From polygons and symbols to polylogarithmic functions, JHEP 1210 (2012) 075, [arXiv:1110.0458].

[48] C. Duhr, Hopf algebras, coproducts and symbols: an application to Higgs boson amplitudes, JHEP 1208 (2012) 043, [arXiv:1203.0454]. 